[Arbeiten des Vereins zur Förderung der naturwissensch. Erforschung der Adria in Wien.]

\title{
Einige Ergebnisse der VII. Terminfahrt S. M. S. Najade im Sommer 1912 in der Adria.
}

\author{
Von \\ Prof. Dr. Adolf Steuer (Innsbruck).
}

Mit 14 Textfiguren.

\section{Phaoplanktonische Stundenbeobachtungen.}

Während der bisherigen Terminfahrten wurden vón den Hydrographen Stunde für Stunde sog. "fliegende Beobachtungen" der Temperatur und des Salzgehaltes des Meerwassers an seiner Oberfläche bei voller Fahrt ausgeführt. Diese ermöglichen wenigstens von der Beschaffenheit des Oberflächenwassers zu verschiedenen Jahreszeiten ein annähernd richtiges Bild zu geben, während für die tieferen Wasserschichten nur Daten aus den an den Querprofilen liegenden "Stationen I. Ordnung" vorliegen, aus denen die thermischen und chemischen Verhältnisse des Tiefenwassers in den verschiedenen Zonen rekonstruiert werden müssen. Bisher war Netzplankton fast ausnahmslos nur längs der Profile gefischt worden. In einem der ersten Berichte über die Terminfahrten ist in Aussicht gestellt worden, es werde nach Beendigung der Fahrten möglich sein, „Karten der Verbreitung der verschiedenen Tierformen am Grunde des Meeres zu entwerfen“. Es zeigt sich, daß nicht einmal für einzelne der gemeinsten Phaoplanktonten halbwegs zutreffende Verbreitungskarten entworfen werden können, wenn nur die auf den Stationen I. Ordnung gewonnenen Daten vorliegen. Ich hatte daher schon bei der vorletzten Terminfahrt (N. VI, Mai-Juni 1912), an der ich zum ersten Male als Zoologe teilnehmen konnte, während der Rückfahrt mit Richards Oberflächennetz stündlich gefischt und mich von der Brauchbarkeit dieser Methode überzeugt. Während der letzten 
Terminfahrt (N. VII, August-September 1912) war ich in der Lage, nahezu ununterbrochen derartige Stundenfänge (166 an der Zahl) auszuführen und sofort an Bord zu untersuchen.

Zur Technik derartiger Stundenbeobachtungen möchte ich kurz folgendes bemerken. Die hydrographischen Beobachtungen werden in $\mathrm{Ab}^{-}$ ständen von $10 \mathrm{SM}$, bzw. einer Fahrstunde gemacht. Es empfiehlt sich wegen der Individuenarmut des südadriatischen Phaoplanktons das Richard-Netz möglichst lange, d. i. ${ }^{3 / 4}$ Stunden fischen zu lassen. So bleibt noch $1 / 4$ Stunden, um im aufgeholten Netz das Wasser ablaufen lassen zu können und nach Entnahme der Planktonprobe Netz und Eimer zu reinigen.

Um die Verbreitung des Phaoplanktons während einer Jahreszeit genauer festzustellen, ist es nötig, das Untersuchungsgebiet möglichst rasch nach vielen Richtungen zu durchfahren. Schon während der III. Terminfahrt (August-September 1911) zeigte sich bei den hydrographischen Beobachtungen, „wie außerordentlich wichtig es ist, um vollkommen vergleichbare Werte zu erhalten, die Stationen der Profile möglichst rasch hintereinander zu absolvieren." In den drei Wochen, die damals seit der Ausreise verflossen waren, hatte sich z. B. das Meerwasser der nördlichen Adria (Profil Ravenna-Lussin) merklich abgekühlt. Bei der Ausreise der VII. Terminfahrt zeigte das Phaoplankton durchaus sommerliche Verhältnisse, während bei der Rückfahrt otwa vom Profil Ancona-I. Incoronata ab (7. IX.) nordwärts bereits das herbstliche Vordringen einiger Hochseeplanktonten zu beobachten war. Selbstverständlich ist bei den Stundenbeobachtungen der Biologe dem Hydrographen gegenüber stets sehr im Nachteil. Er wird sich immer die Frage vorzulegen haben: Kommt ein Planktont dort wirklich nicht vor, wo er nicht gefangen wurde? Dazu sind die hydrographischen wie die Planktonverhältnisse in einem so schmalen Meeresteil einem oft von Stunde zu Stunde sich fühlbar machenden Wechsel unterworfen. Gerade in solchen Fällen kann sich allerdings ein intensives Zusammenarbeiten des Hydrographen und Biologen äußerst fruchtbringend gestalten. Unangenehm bemerkbar macht sich bei biologischen Stundenbeobachtungen die vertikale Wanderung des Planktons, mit der bei der Auswertung der gefundenen Daten immer zu rechnen ist. Glücklicherweise wurden bei den Terminfahrten die Profilarbeiten größtenteils am Tage erledigt, und die Fahrten zum nächsten Profil erfolgten bei Nacht; dadurch erhielt ich aus den Vertikal-Schließnetzfängen an den Profilstationen das gesamte Netzplankton und konnte während der anschlieBenden Nachtfahrt mit dem Richard-Netz auch noch auf den Fang wenigstens eines 
Teiles der nachts aus tieferen Schichten aufsteigenden Planktonten rechnen.

Aufgabe der Terminfahrten war zunächst die Erforschung der adriatischen Hochsee. Die Phaoplanktonverteilung in dem dalmatinischen Inselgewirr konnte daher nur bei gelegentlichem Passieren der Kanäle

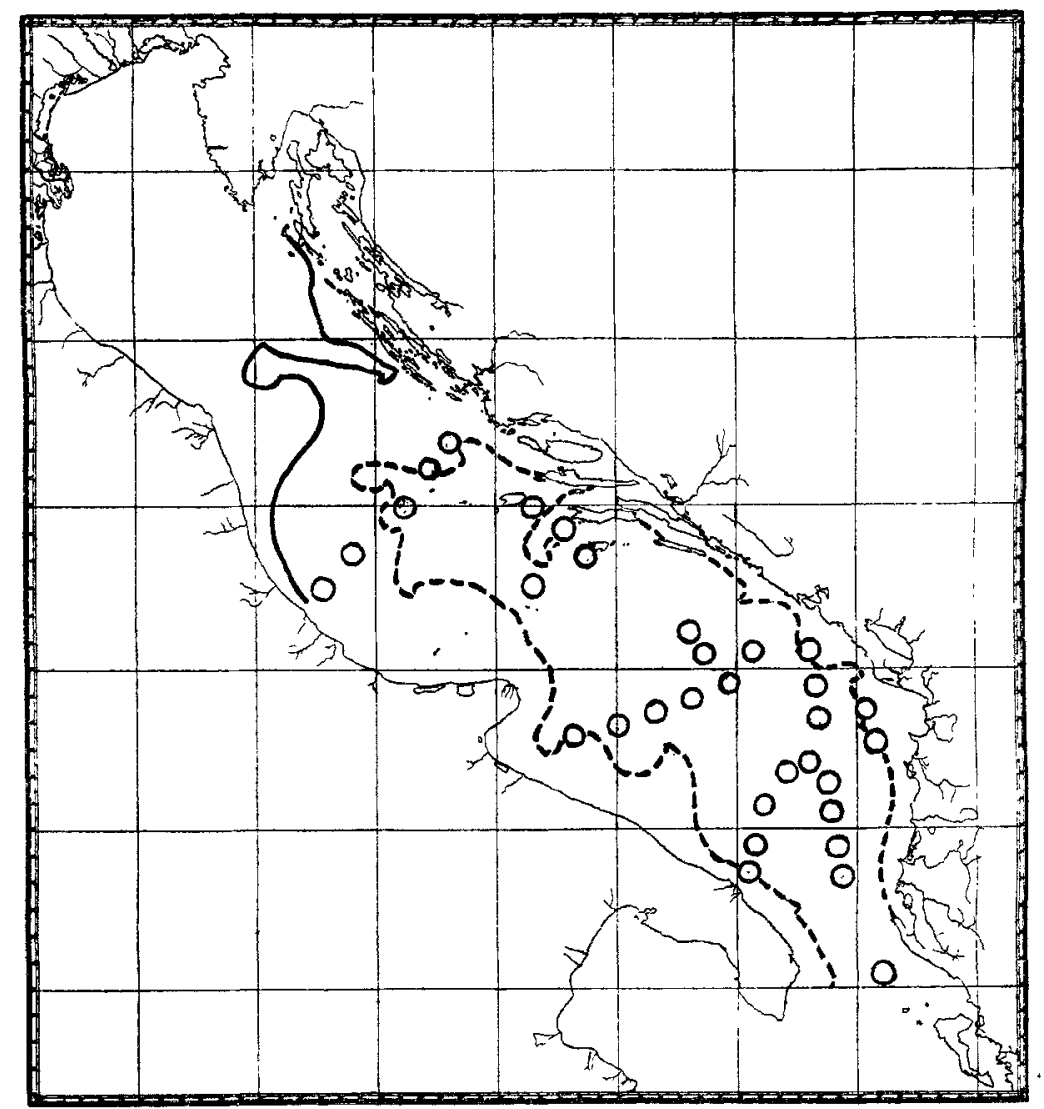

Fig. 1. Verbreitung Koloniebild. Radiolarien in der Adria im Aug.-Sept, 1912 (N. VII). Isohaline $37 \%$. -...- Isohaline $38 \%$.

ganz unvollständig beobachtet werden. Soviel ließ sich feststellen, daß echte Hochseeformen oft in gewaltigen Mengen in diese Kanäle entführt werden (so bei N. VII Creseis im Kanal von Curzola). Demnach muß nahezu reines Hochseewasser zeitweilig die Kanäle erfüllen, während andererseits Küstenwasser mit typisch neritischen Planktonten (vielfach von der Ausmündung der Karstflüsse weg) weit auf die Hochsee hinausgeführt werden kann (bei N. VII Außenküste von I. Grossa). 
Den Küsten fremder Staaten darf sich die "Najade" nur bis auf 10 SM nähern. Glücklicherweise verläuft die Grenze des Hochsee- und Küstenplanktons zumeist 10 bis $20 \mathrm{SM}$ von der Küste entfernt. Nur in der Südadria verschmälert sich der Gürtel des neritischen Planktons offenbar wegen des steileren Küstenabfalles derart, daß hier eine

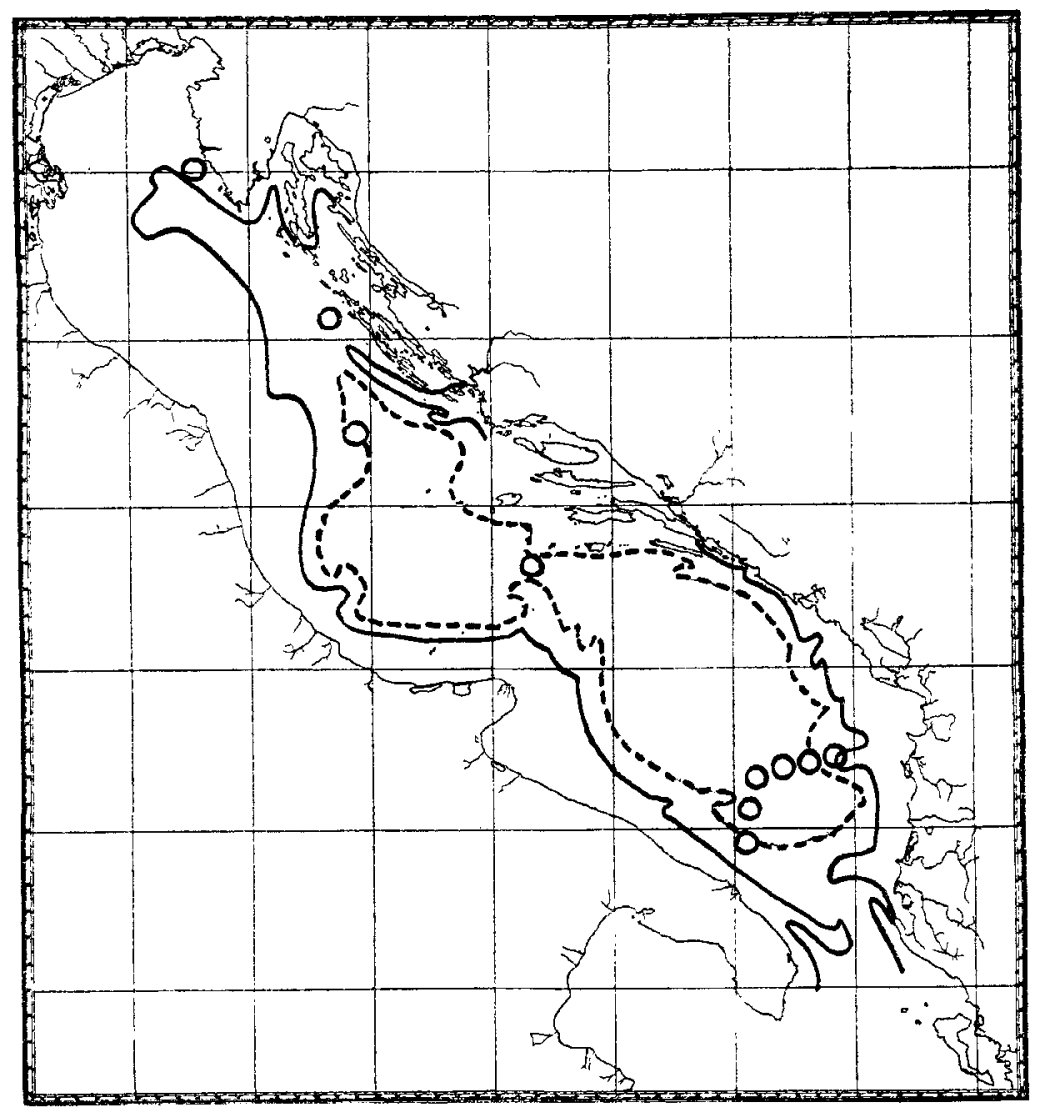

Fig. 2. Verbreitung Koloniebild. Radiolarien in der Adria im Mai-Jumi 1912 (N. VI). Isohaline $37 \%$. -...-- Isohaline $38 \%$.

gröBere Annäherung an die Küste nötig wäre, um auch in diesem Gebiet die genaue Grenze des Hochsee- und Küstenplanktons feststellen zu können.

Als ein Beispiel ausgesprochen ozeanischer Planktonten mögen zunächst die koloniebildenden Radiolarien in ihrer sommerlichen Ausbreitung im Adriabecken besprochen werden. Ein Blick auf die Verbreitungskarte (Fig. 1) lehrt, daß die Mehrzahl der Fänge innerhalb 'der 
$38 \%$-Isohaline ${ }^{1}$ ) liegt, die ja von Grund als Grenze des Hochseewassers angegeben wird; ${ }^{2}$ ) nur wenige liegen außerhalb dieser Linie, keine einzige Fundstelle aber überschreitet die $37 \%$-Isohaline. Es ist sehr bezeichnend, daB die drei nördlichsten Fundstellen an der dalmatinischen Küste auf der Heimreise verzeichnet wurden (herbstliches Vor-

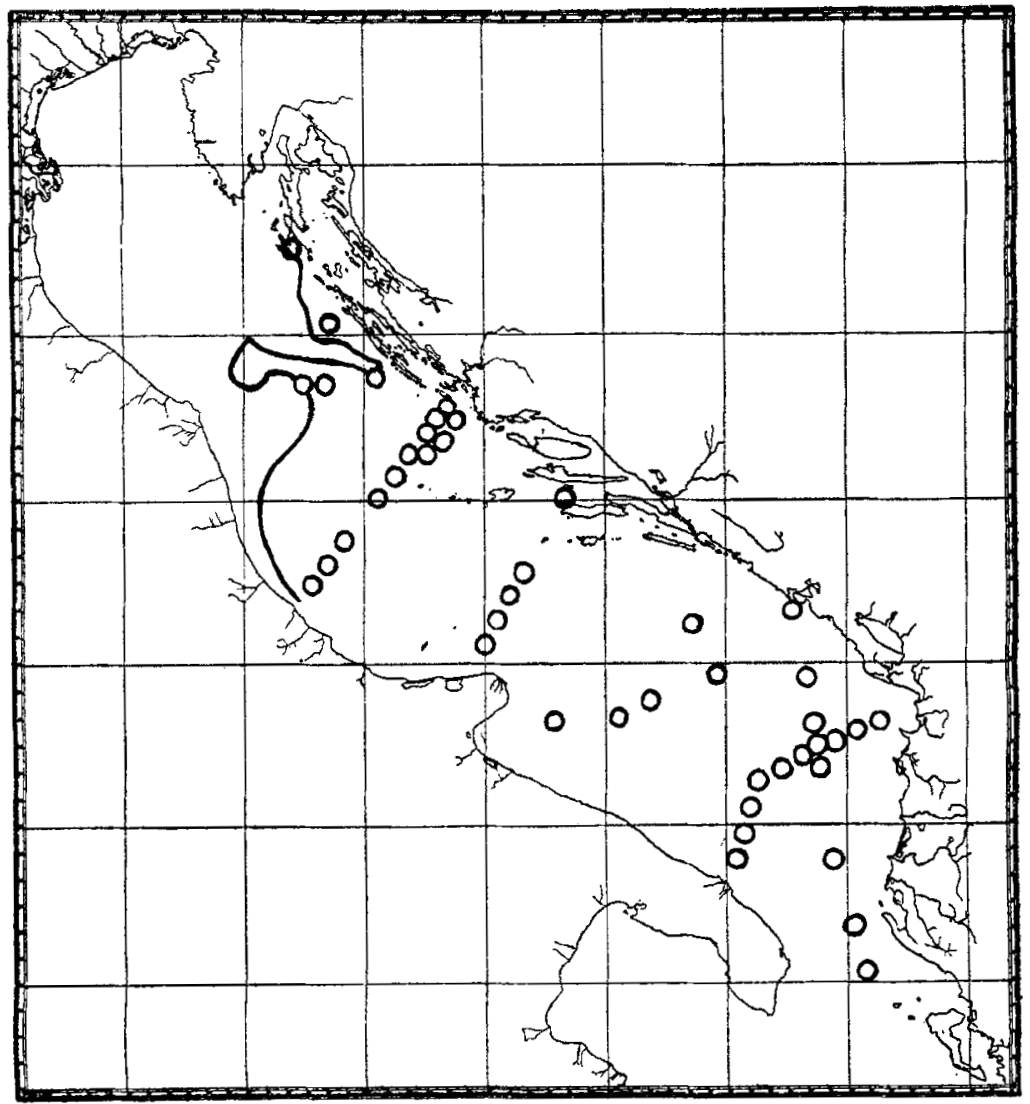

Fig. 3. Verbreitung der Halocypriden in der Adria in Aug.-Sept. 1912 (N. VII). Isohaline $37 \%$.

dringen der Hochseeformen). Auch während der Frühlingsfahrt (N. VI, Fig. 2) begrenzen die $38 \% 0^{-} \mathrm{bzw}, 37 \%$-Isohaline die Vorkommnisse der koloniebildenden Radiolarien. Entsprechend der größeren Ausbrei-

1) Die hydrographischen Daten stellte mir in liebenswürdiger Weise der Chef der ozeanographischen Arbeiten, Prof. A. Grund (Prag) zur Verfügung, wofür ich ihm auch an dieser Stelle herzlich danke.

2) Mitt. d. geogr. Gesellsch. zu Wien 1911, S. 11 d. Sep. 
tung des Küstenwassers an der italienischen und albanesisch-dalmatinischen Küste liegen aber die Fundstellen viel mehr in der küstenfernen, zentralen Adria, und da das Hochseewasser im Frühjahr noch bis an die istrianische Halbinsel heranreicht, liegen auch die nördlichsten Fundorte noch viel weiter nördlich als zur Sommerszeit.

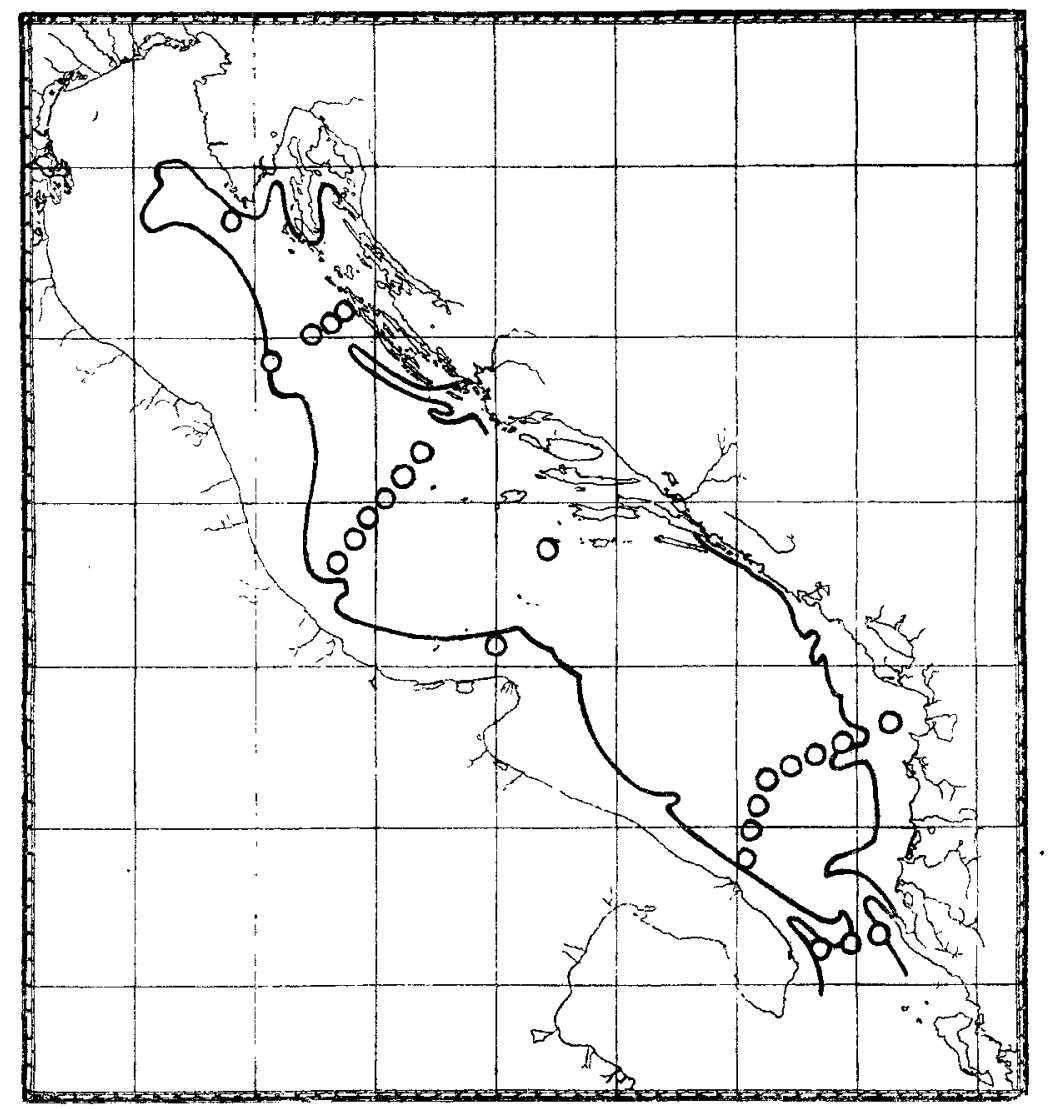

Fig. 4. Verbreitung der Halocypriden in der Adria im Mai-Juni 1912 (N. VI).

Isohaline $37 \%$.

In ihrem maximalen Vorkommen schon weit weniger an das starksalzige Hochseewasser gebunden erscheinen die Halocypriden ${ }^{1}$ ), die aber, nebenbei bemerkt, mit dem Richard-Netz zumeist nur zur Nachtzeit gefangen werden. Bei Tage halten sie sich gewöhnlich unterhalb $20 \mathrm{~m}$ auf; gegen 4 bis $5^{\text {h }}$ nachmittags beginnt der Aufstieg, nach Mitternacht Tiefen vor. 
(selten schon gegen Sonnenuntergang) ist die Oberfläche erreicht. In den ersten Morgenstunden schon setzt der Abstieg ein.

Die. 37\% Grenze, (Fig. 3), die nur an zwei Stellen im Frühjahr 1912 (Fig. 4) überschritten wurde.

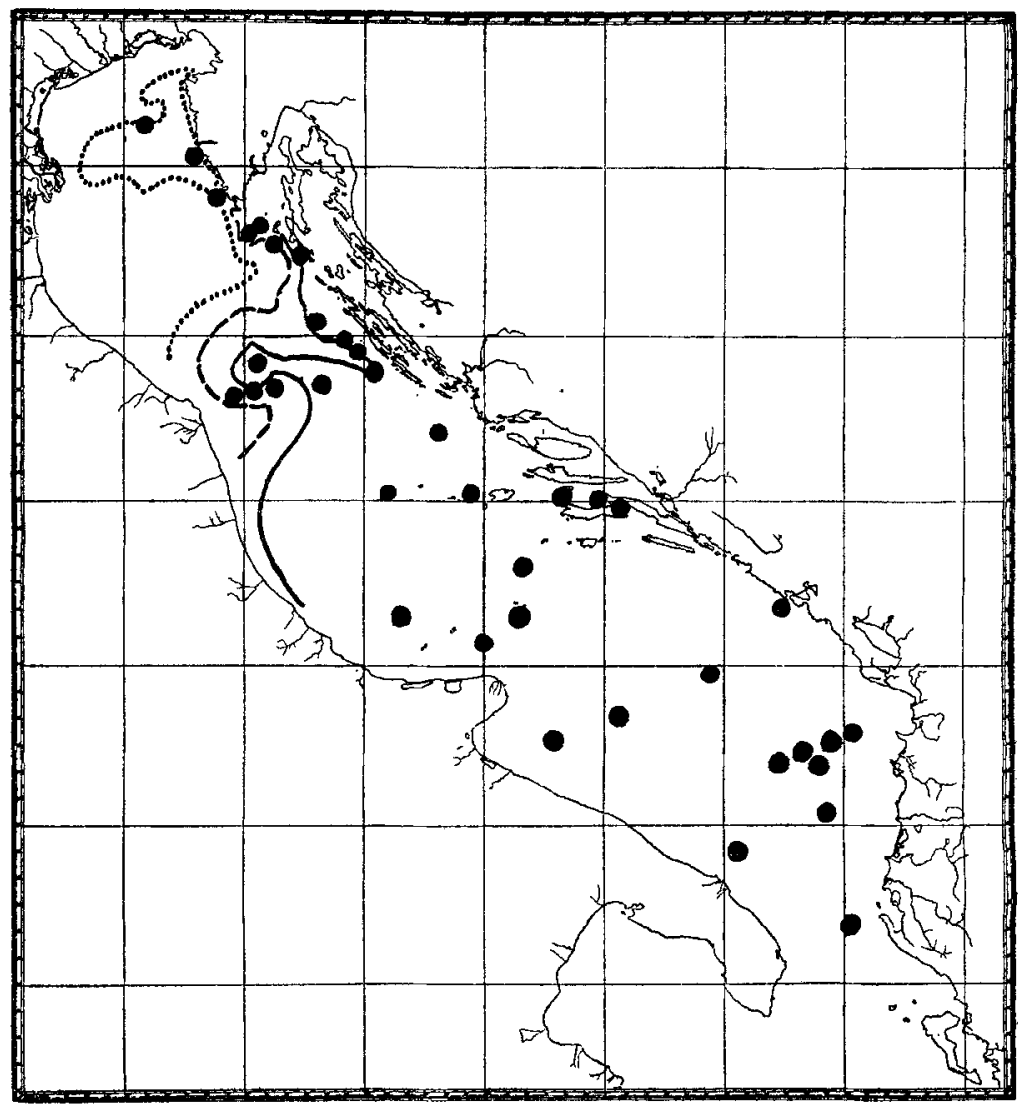

Fig. 5. Verbreitung der Gattung Copilia in der Adria im Aug.-Sept. 1912 (N. VII). Isohaline $37 \%$. ----- Isohaline $36 \%$ \% Isohaline $35 \%$.

Ein Vergleich der Kartenskizzen Fig. 1 und 3 zeigt aber auch, daß die Halocypriden viel weiter an die Nordgrenze der für sie kritischen Isohaline herankommen als die koloniebildenden Radiolarien. Bezeichnenderweise wurden auch bei den Ostracoden während der Sommerfahrt (das gleiche gilt auch für die Orbulinen) die nördlichsten Fundstellen wieder erst auf der Heimfahrt notiert, während umgekehrt im Frühling auf der Rückreise von der Nordspitze der I. Grossa an keine. 
Ostracoden mehr beobachtet wurden (sommerlicher Rückzug der Hochseeplanktonten).

Noch mehr euryhalin ist das Genus Copilia (Fig. 5), als dessen Nordgrenze im Sommer die $36 \%_{00^{-}}$, ja vielleicht sogar die $35 \%$-Isohaline angenommen werden kann. Allerdings dürfte die „Vorhut" dieser Cope-

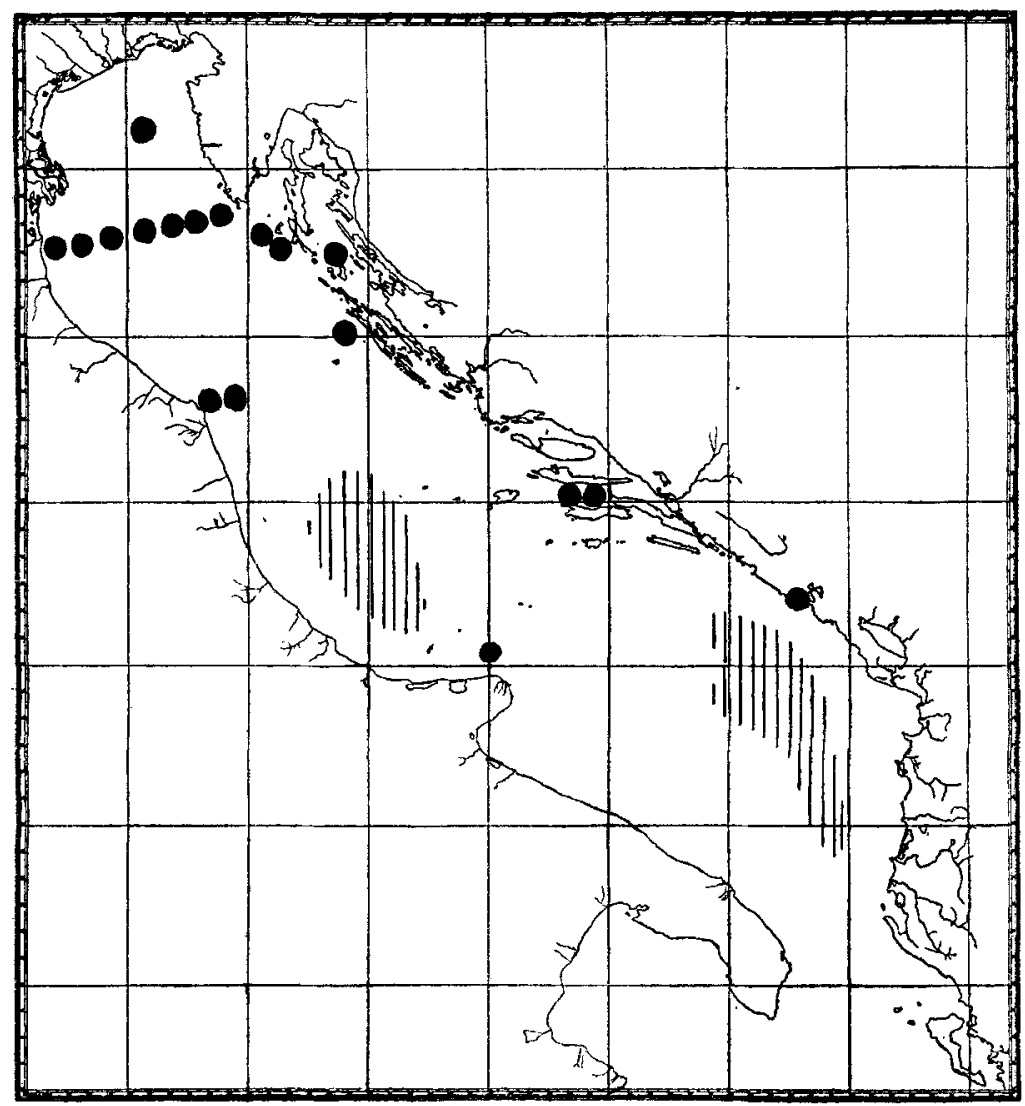

Fig. 6. Verbreitung der Cladoceren in der Adria im Aug.-Sept. 1912 (N. VII). Podon.

Ill Evadne-armes Gebiet.

poden-Gattung gröBtenteils aus Jugendstadien und Weibchen bestehen; Männchen notierte ich nur im Bereiche der 37\%o-Isohaline.

Uber die ganze Adria verbreitet ist endlich das Cladoceren-Genus Evadne. Auffallend ist nur, daß sich im nördlichen wie im südlichen Becken Gebiete finden, in denen Evadne selten war oder fehlte (Fig. 6). Ein ähnliches Evadne-armes Gebiet fand sich auch im Frühling 1911 und 1912 (Fig. 7). (Für das Südbecken liegen leider von den Fahrten 
N. II und N. VI zu spärliche Daten vor, da nur an den Profilen gefischt worden war.) Vielleicht ist es der hohe Salzgehalt $(>38 \%$, der in den Evadne-armen Gebieten die gleichmäBige Ausbreitung der Tiere über die ganze Adria unterbricht. Nach Apstein (Cladocera, Bull. trimestr. I. Partie 1910) wurde nämlich in den Nordmeeren häufigeres

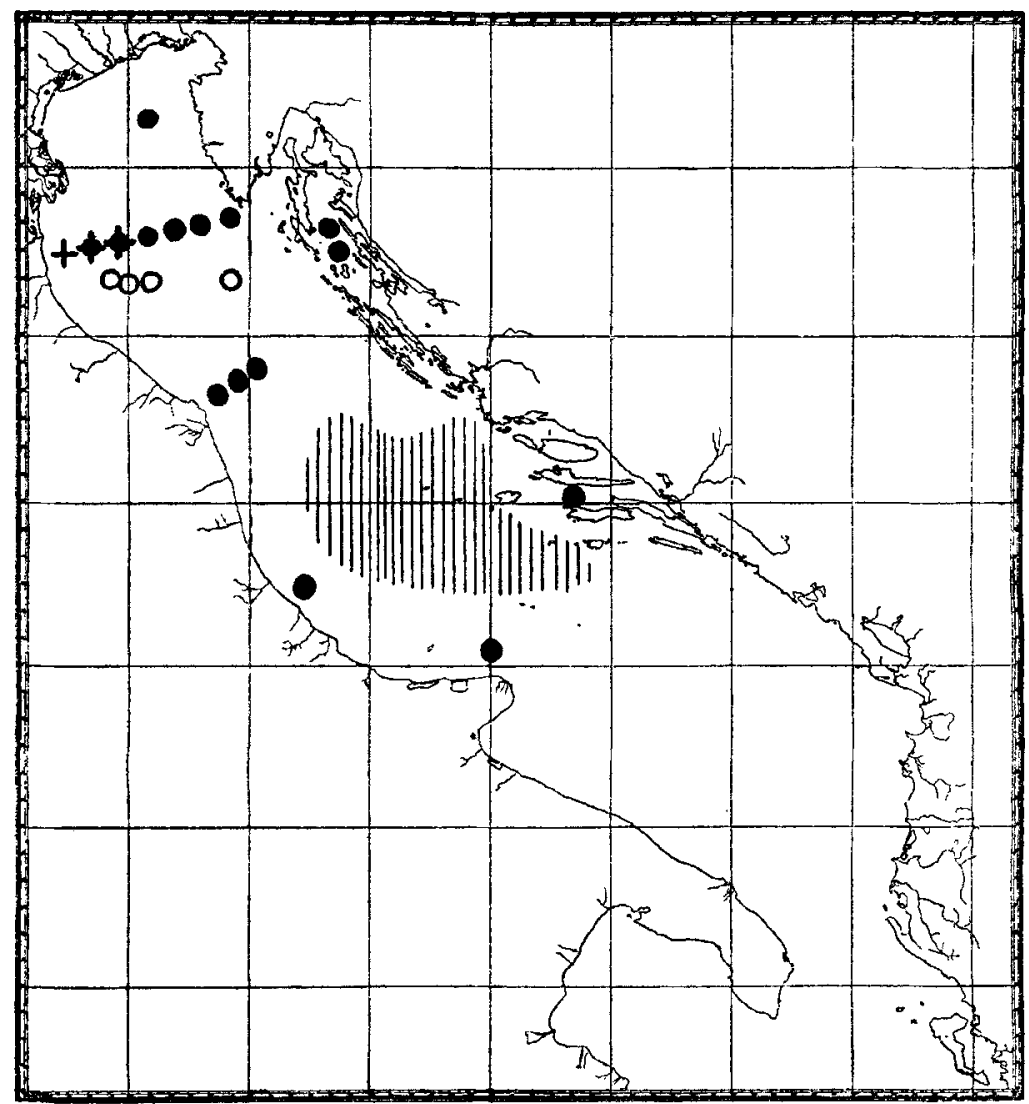

Fig. 7. Verbreitung der Cladoceren in der Adria.

O Podon im Mai-Juni 1911 (N. II). + Podon im Febr. 1912 (N. V).

Podon im Mai-Juni 1912 (N. VI). |l| Evadne-armes Gebiet in maximaler Ausdehnung im Frühjahr 1911 und 1912.

Vorkommen der Evadne spinifera, der gemeinsten Cladocere in der Adria, bei ca. 15-35\% Salzgehalt beobachtet. „In der Sargassosee lebt sie bei 36-37\% Salzgehalt." Evadne mit Dauereiern notierto ich fast ausnahmslos in der Nähe des Festlandes bzw. der Inseln (Pelagosa, Cazza, Lesina. Lissa, Pomo). Wesentlich anders ist das Verbrei- 
tungsgebiet der adriatischen Podon-Arten, die aber bei den Stundenbeobachtungen fast nie erbeutet wurden, da sie bekanntlich etwas tiefere Wasserschichten bewohnen.

Weit verbreitet fanden sich endlich unter den Heteropoden die Atlantiden und unter den Pteropoden das Genus Creseis, denen nur

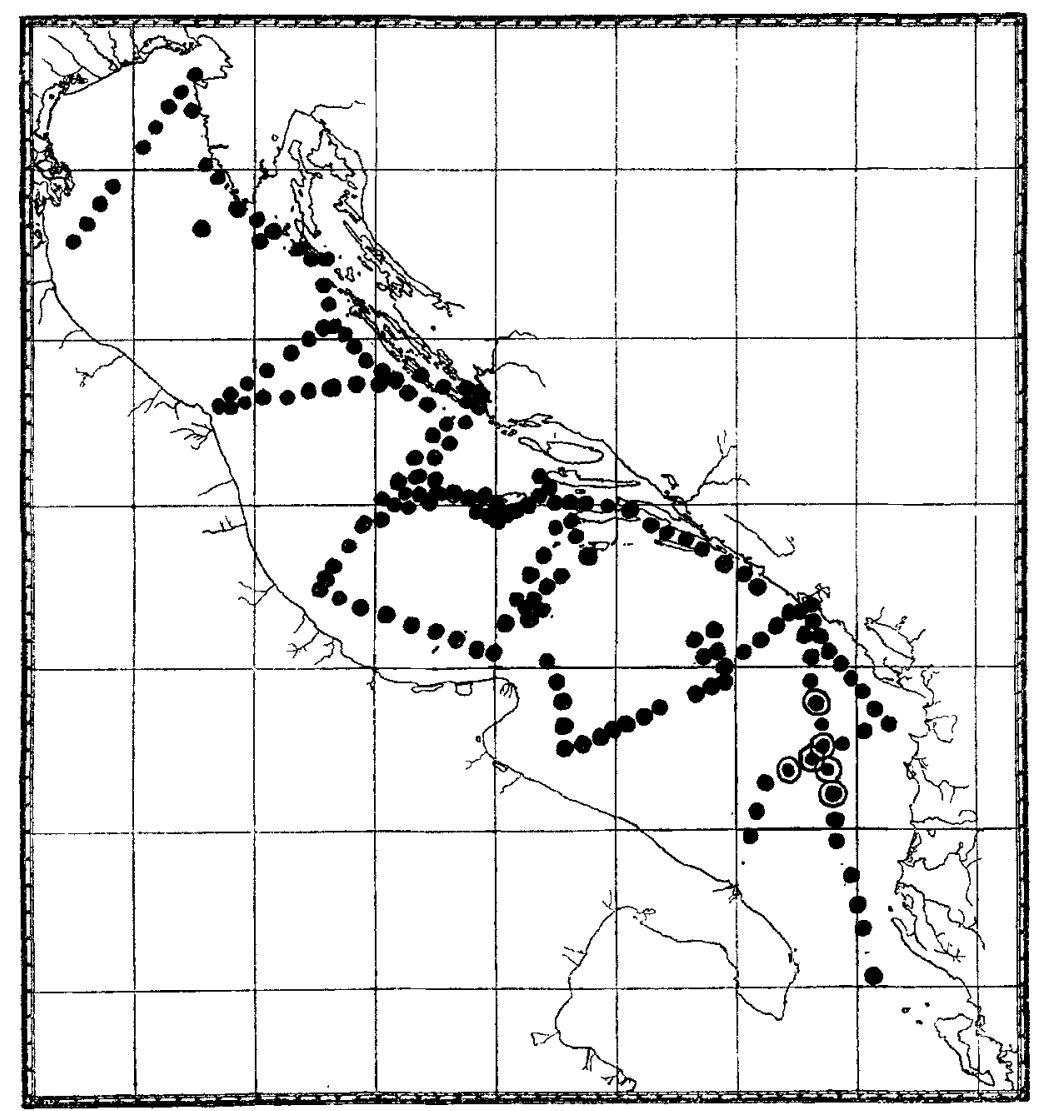

Fig. 8.

Verbreitung zweier Pteropoden-Gattungen in der Adria im Aug.-Sept. 1912 (N. VII). Creseis.

O Clio.

das stark ausgesüBte Wasser in der Gegend des Ravenna-Profils nicht mehr zu behagen schien (Fig. 8). Nur auf den zentralen Teil des südlichen Beckens blieb eine zweite Pteropoden-Gattung (Clio) beschränkt, deren Verbreitungsgebiet von der "Najade" zweimal durchfahren worden war. 


\section{Verbreitung einiger neritischer Planktonten.}

In ihrer Ausbreitung eng an das seichte Küstenwasser gebunden erscheinen die meisten neritischen Planktonten der Adria. An der italienischen Küste überschreiten sie selten eine 10-20 SM breite Zone. Nur in der nordadriatischen Flachsee vergröBert sich ihr Wohngebiet

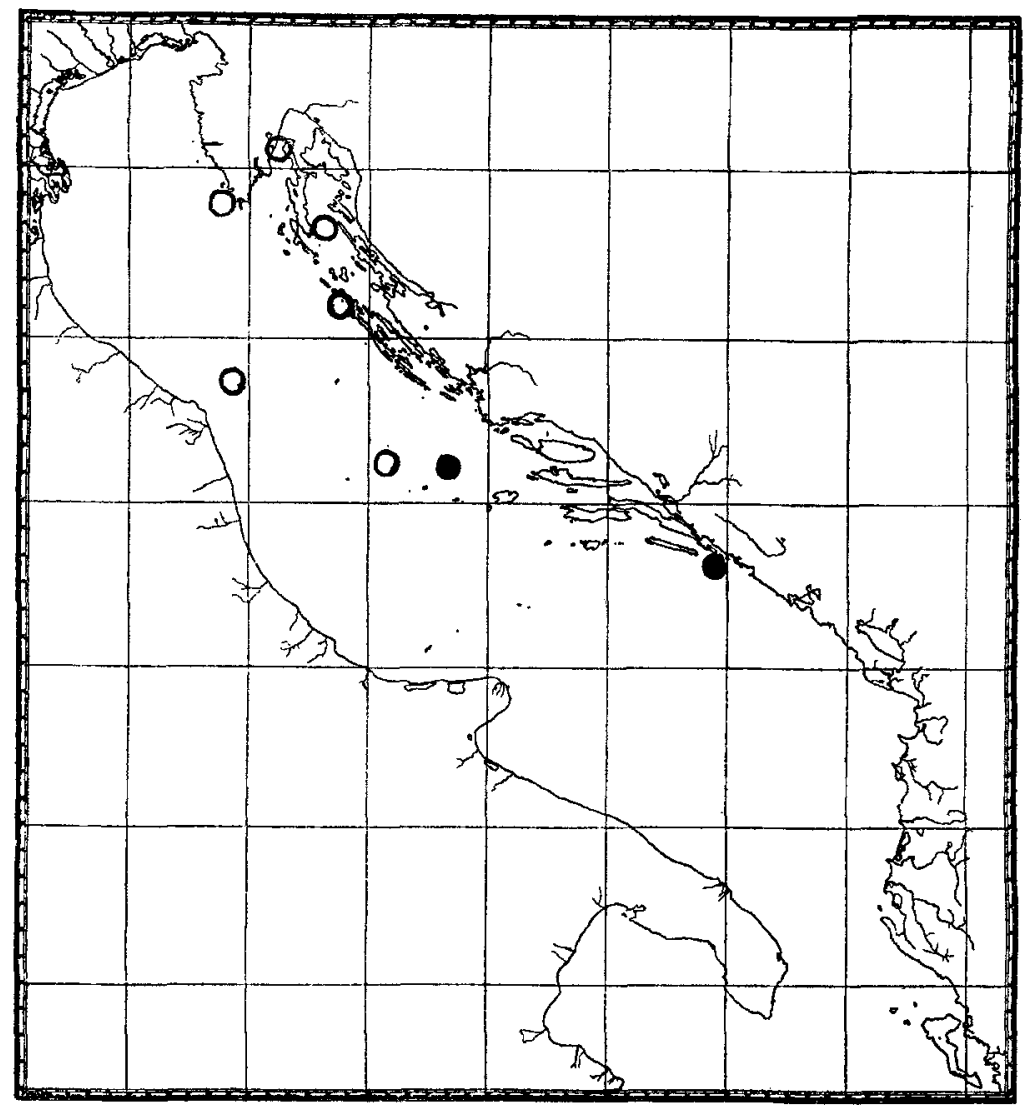

Fig. 9. Verbreitung der Stomatopoden-Larven in der Adria im Frühling. O Mai-Juni 1912 (N. VI).

Mai-Juni 1911 (N. II).

bedeutend, während es sich an den Steilküsten im Süden entsprechend verengt. Recht lehrreich ist die Ausbreitung der schon erwähnten Cladoceren-Gattung Podon. Im Material der Februarfahrt 1912 (N. V) fand ich sie bisher nur an der italienischen Seite des Ravennaprofiles (Fig. 7); von da breitet sie sich im Frühjahr und Sommer an der italienischen Küste aus (Fig. 6). An den Steilküsten der Südadria kommt 
Podon vielleicht nur innerhalb der 10 SM-Zone vor, die, wie erwähnt, unser Schiff nicht überschreiten durfte. Nyctipelagische Küstenformen sind z. B. die Cumaceen, die während der VII. Terminfahrt in der Nordadria und im Kanal von Curzola erbeutet worden waren. Unter den' Ectoparasiten der Zooplanktonten fiel mir auf, dab Microniscus-ähn-

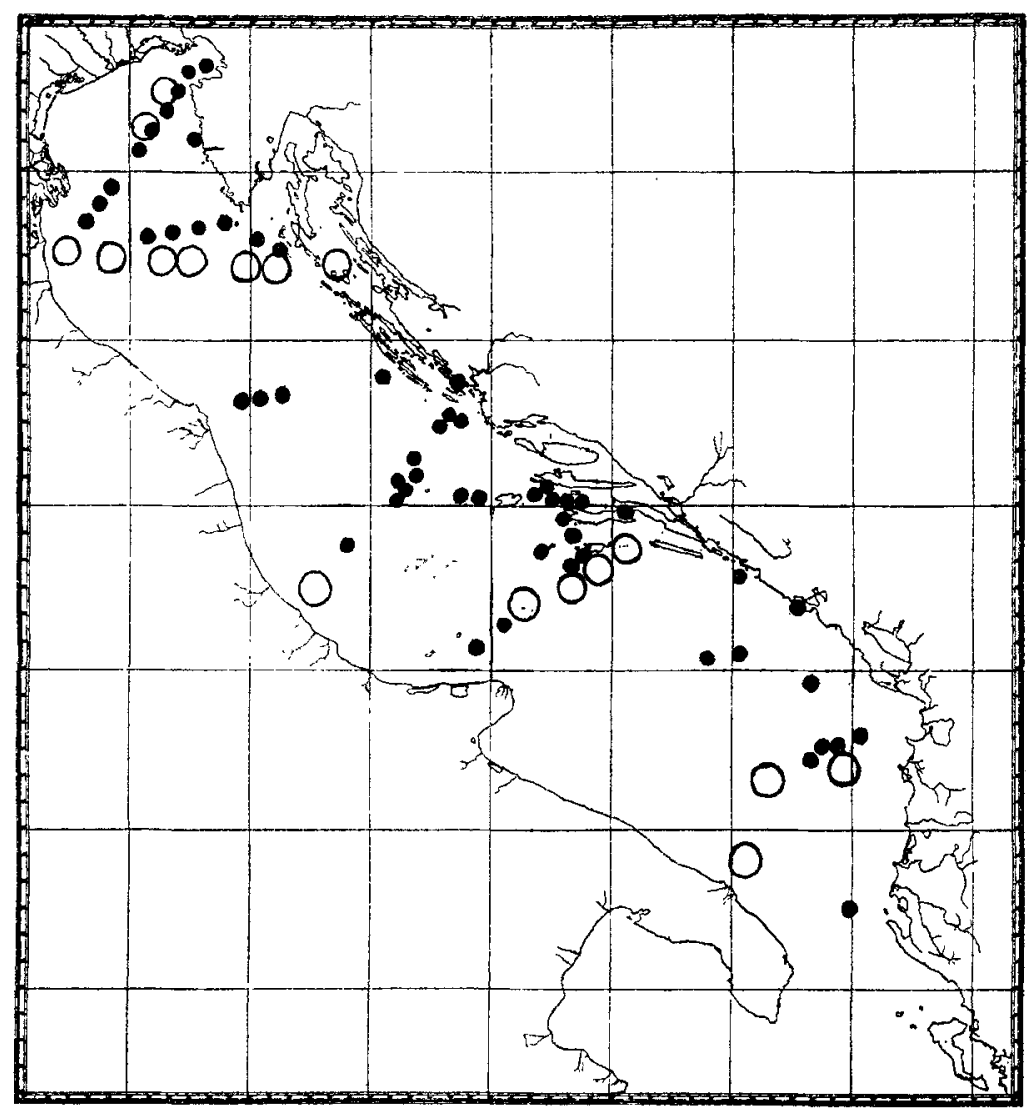

Fig. 10. Verbreitung der Stomatopoden-Larven in der Adria im Sommer. Aug.-Sept. 1912 (N. VII). O Aug.-Sept. 1911 (N. III).

liche Isopoden ebenfalls nur in Küstennähe zur Beobachtung kamen, während die auf Euchaeta hebes lebende Suctorie Tokophrya steueri schon während der Maifahrt auch mitten auf der Hochsee gefunden worden war. Die in Sagitten schmarotzenden, kleinen Trematoden fand ich bisher nur in der nördlichen Adria und im Frühjahr einmal in der südlichen Adria (Station 32). 
Die pelagischen Larven der Benthostiere kommen gröBtenteils nur an der Küste und im Bereiche der Inseln vor, so Actinotrocha, die in 20-30 m Tiefe leben dürfte, ferner Tornaria, Echinospira, Pilidium u.v.a. Stomatopodenlarven fand ich während der Frühlingsfahrt (N. VI) noch ziemlich eng auf das Küstengebiet der Nordadria beschränkt, und zwar wurden sie ausnahmslos erst während der Rückreise (vom 9.-12. VI) erbeutet (Fig. 9). Gegen den Sommer zu breiten sie sich über das ganze Gebiet aus und fehlen selbst der südadriatischen Hochsee nicht (Fig. 10).

\section{Das Plankton im nördlichen und südlichen tiefen Becken.}

Eine Frage für sich ist die Verteilung der Planktonorganismen im nördlichen und südlichen tiefen Becken. Das nördliche, bis $260 \mathrm{~m}$ tiefe Pomobecken steht durch die ca. $180 \mathrm{~m}$ tiefe Pelagosarinne mit dem bis zu $1132 \mathrm{~m}$ abfallenden südlichen Adriabecken in Verbindung. Es gibt nun, wie ich aus meinen bisherigen Beobachtungen schließen möchte, Planktonten, die beide Gebiete bewohnen und in ihrer Ausbreitung an die $150 \mathrm{~m}$ Isobathe gebunden sind, obwohl sie zeitweilig wenigstens in so hohen Wasserschichten schweben, daß eine Ausbreitung ins seichtere Wasser leicht möglich wäre.

So wurden Aulacanthiden einmal oberflächlich noch in einem Schließnetzfang von $20-60 \mathrm{~m}$ Tiefe gefangen, und sie kommen von da ab bis in die größten Tiefen hinab vor. Im Frühjabr 1912 fand ich sie auch im zentralen Pomobecken, im Sommer 1912 dagegen waren sie von dort bereits bis zur Pelagosaschwelle zurückgedrängt (Fig. 11). Lucifer dagegen, der einmal um $6^{\mathrm{h}}$ früh auch mit dem Richard-Netz gefangen wurde, konnte auch im Sommer für das Pomobecken nachgewiesen werden.

Nach der Auffindung der „Zackeneier" läBt sich folgendes über das Laichgeschäft eines Tiefseefisches (Macrurus, nach Fage M. coelorhynchus "une forme d'eau très profonde!") aussagen (Fig. 12): Die Hauptlaichzeit fällt in die Wintermonate, doch ist es jedenfalls sehr auffallend und, wie ich glaube, noch unbekannt, daB sich das Laichgeschäft über das ganze Jahr ausdehnt. Im Materiale von N. V (Febr. bis März 1912) fand ich über 20 Eier nur an den Stationen 11, 13, 14 und 15 des Ortonaprofiles im Pomobecken. Die meisten Eier (davon 8 mit dem Richard-Netz!) wurden auf Station 13 gefangen, wo auch im Frühjahr und Sommer noch Eier gefunden wurden. Dort ist aber zugleich die tiefste Stelle des Pomobeckens. Leider ist namentlich über 
das Winterplankton des südlichen Adriabeckens noch viel zu wenig bekannt, als daB sich über die dortigen Macrurus-Laichplätze etwas aussagen liebe. Es wird ferner noch zu prüfen sein, ob auch in der Adria die Macruruseier in ihrem Vorkommen an Wasser unter $15^{\circ} \mathrm{C}$ gebunden sind.

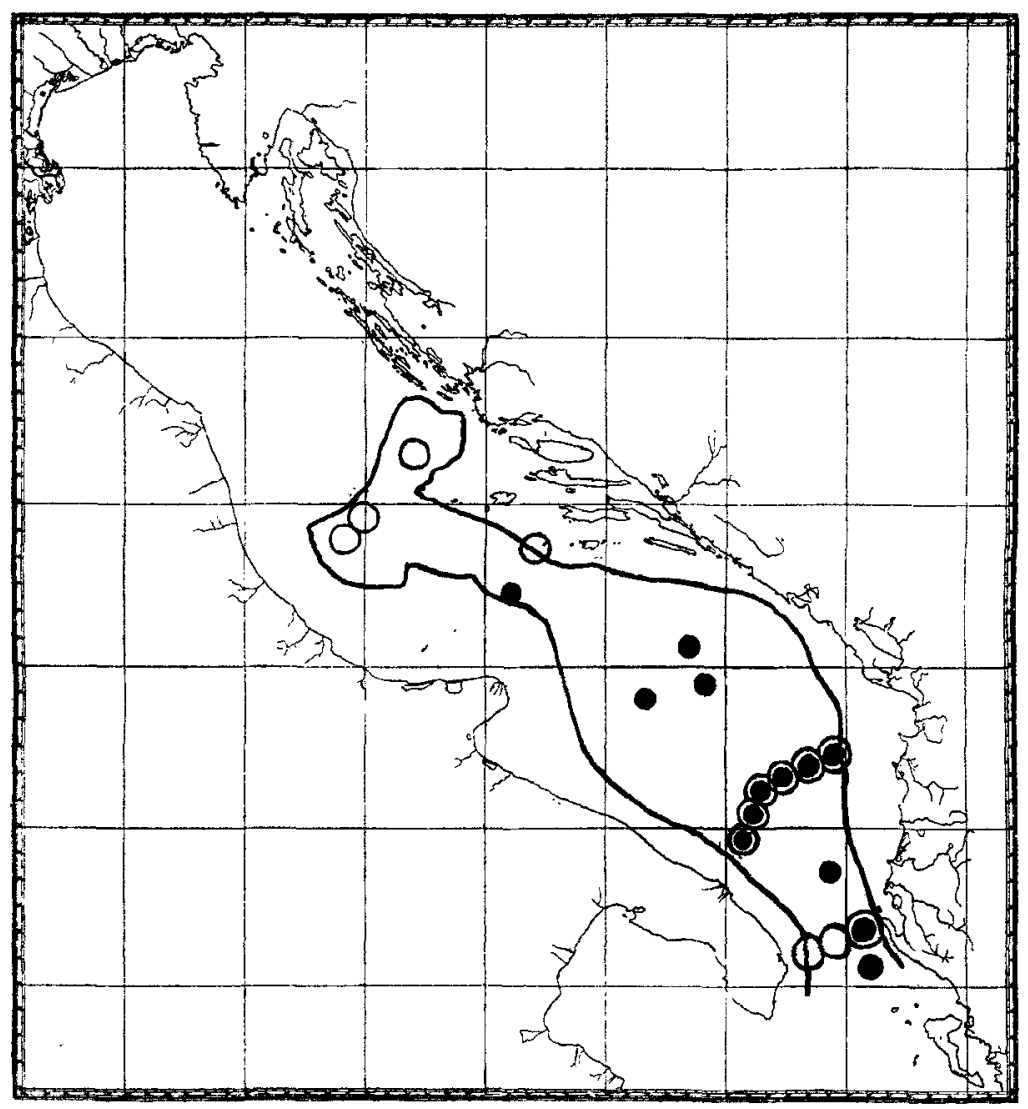

Fig. 11. Verbreitung von Aulacantha in der Adria.

Aug.-Sept. 1912 (N. VII). O Mai-Juni 1912 (N. VI). — $150 \mathrm{~m}$ Isobathe.

AusschlieBlich auf das südliche tiefe Becken beschränkt zu sein scheinen die großen Phaeodarien (Coelodendrum, Aulatractus) und von den Nasselarien das große Litharachnium tentorium Haeckel $^{1}$ ). Dabei fällt auf, daß dieses Radiolar von mir bisher nur im

1) Die adriatische Form stimmt weit besser mit der von Jörgensen (1905) gegebenen Beschreibung überein als mit der Originaldiagnose Haeckels (1862). Der Schalenrand ist immer hutkrempenartig umgebogen. Dieses auffallende Ra- 
tiefsten Teil (Brindisiprofil, z. B. Station 28, 29) gefunden wurde, während die erwähnten Phaeodarien ein wesentlich größeres Areal zu bewohnen scheinen (Brindisiprofil, z. B. Stationen 26-31), und auch in ihrer vertikalen Verteilung von etwa $50-900 \mathrm{~m}$ Tiefe weniger anspruchsvoll sein dürften als Litharachnium, das nur Zonen von etwa

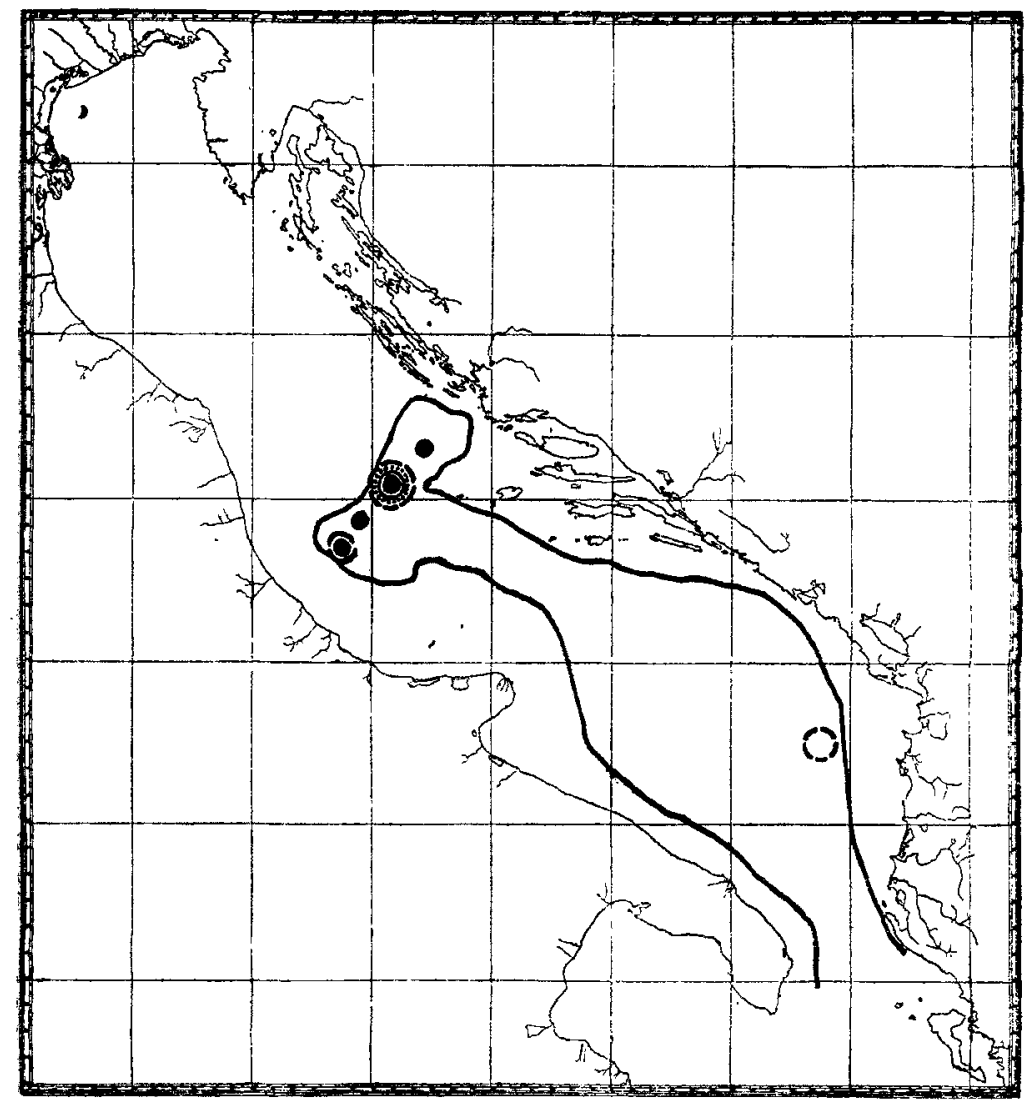

Fig. 12. Verbreitung der Macrurus-Eier in der Adria.

- Febr.-März 1912 (N. V).

O Mai-Juni 1911 (N. II).

O) Aug.-Sept. 1912 (N. VII).

Ang.-Sept. 1911 (N. III).

C Nov.-Dez. 1911 (N.IV).

150-500 m Tiefe zu bewohnen scheint (Fig. 13). In ihrer horizontalen Ausbreitung stimmen die großen Phaeodarien genau mit einer Meduse

diolar ist bisher nur aus dem Mittelmeer (Messina, Neapel) und aus den Nordmeeren (zwischen den Faroerinseln und Norwegen, sowie aus Norwegischen Fjorden) bekannt. 
Aegineta flavescens überein, die das Südbecken von etwa $300 \mathrm{~m}$ Tiefe abwärts bewohnt. Nur nachts fanden sich größtenteils kleinere Individuen in Schließnetzfängen schon von $20-60 \mathrm{~m}$ Tiefe an. Die skotoplanrtonischen Fische endlich (Chauliodus, Stomias, Cyclothone, Omosudis u. a.) sind in ihrem Vorkommen größtenteils auf

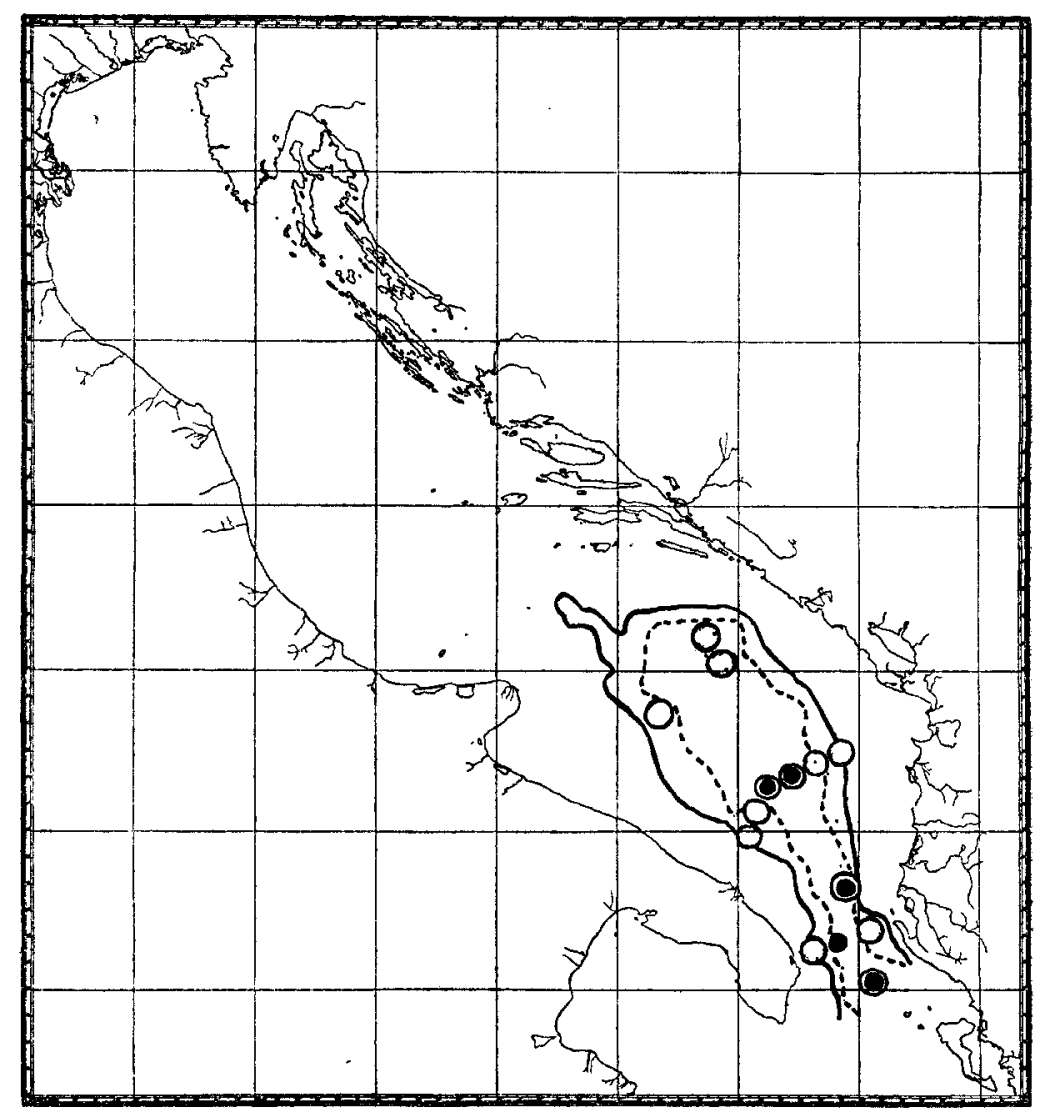

Fig. 13. Verbreitung einiger Radiolarien in der südlichen Adria.

große Phaeodaria.

Litharachnium.

$400 \mathrm{~m}$ Isobathe.

den zentralsten Teil des tiefen Beckens, den die $800 \mathrm{~m}$-Isobathe begrenzt, beschränkt. Nur zur Nachtzeit steigen auch diese Tiefseefische (allerdings wieder zumeist in jüngeren Exemplaren) gegen die Oberfläche empor. Genauere Angaben, in welcher Tiefenzone jede Art im Maximum vorkommt, werden sich erst machen lassen, wenn die mit den Jungfischtrawl-Serienfängen erbeuteten Tiere gezählt sein werden. Vor- 
läufig habe ich nur aus einigen dieser Fänge die Amphipoden ausgesucht und gezählt.

Fig. 14 zeigt die vertikale Verteilung einiger Amphipodengattungen in der Südadria zur Nachtzeit (das unterste Netz aus etwa $900 \mathrm{~m}$ kam

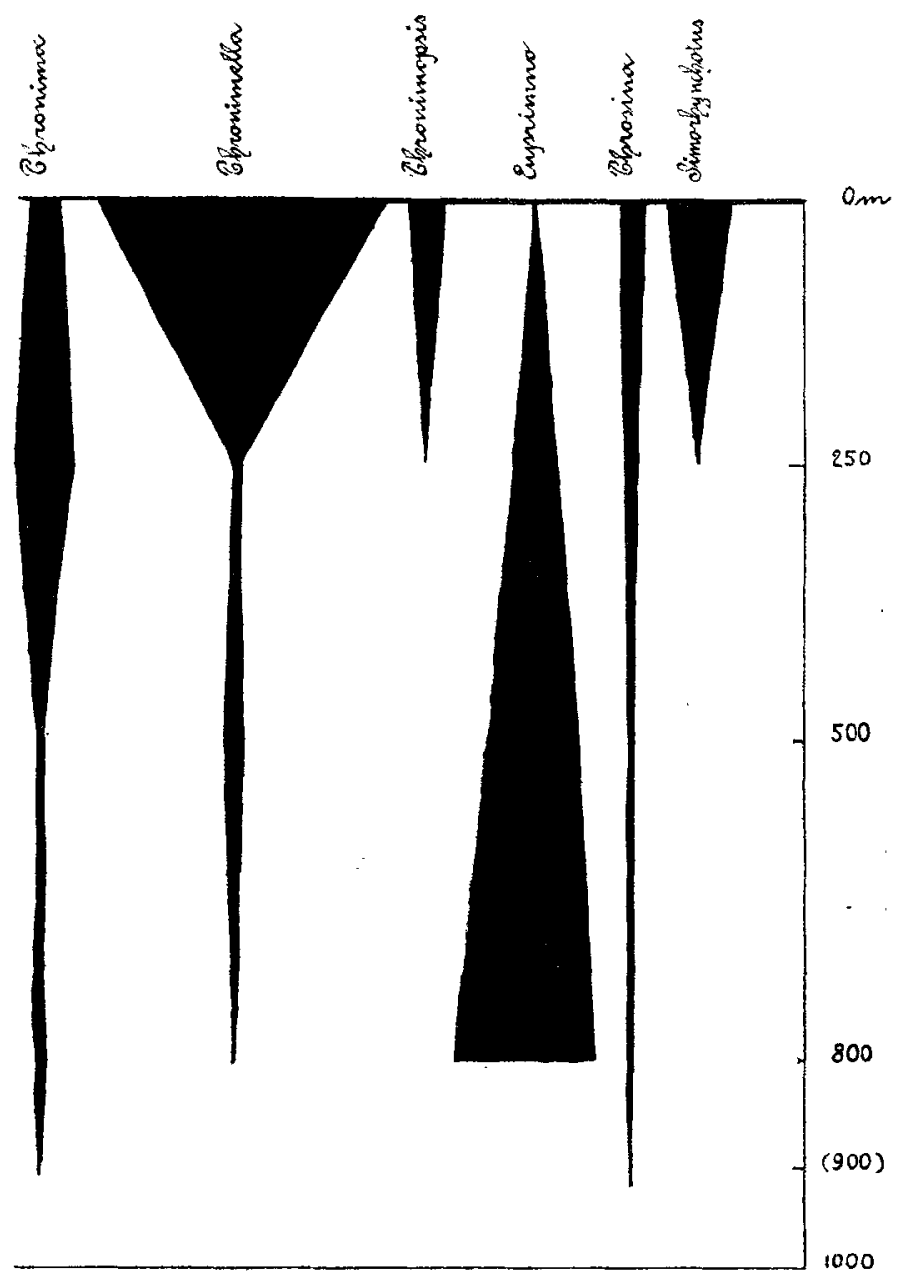

Fig. 14. Vertikale Verteilung einiger Amphipodengattungen in der sũdl. Adria auf Grund eines Jungfischtrawl-Serienfanges (N. VII, $\mathrm{IT}_{4}$ ) in der Nacht vom 29., 30. VIII. 1912.

leider leer herauf; Fehlfang?). Man sieht, daß tatsächlich, wie schon früher behauptet worden war, viele Amphipoden pamplanktonisch sind und in größerer Volksstärke die Zone von 0 bis etwa $300 \mathrm{~m}$ bewohnen. Nur bei einer Form nahm gegen die Tiefe die Quantität kontinuierlich zu. 


\section{Allgemeine Resultate.}

Die Jungfischtrawl-Serienfänge, die während der beiden letzten Terminfahrten nach der Hjortschen Methode in systematischer Weise ausgeführt worden waren, haben uns mit einem ungeahnten Artenreichtum des adriatischen Tiefenplanktons bekannt gemacht. Fast alle der gefundenen Tiefseefische, Heteropoden. Appendicularien, Crustaceen usw. sind für die Adria neu. So ist z. B. die Zahl der seit der "Virchow"-Fahrt (1909) aus der Adria bekannten planktonischen Amphipodengattungen auf das doppelte angewachsen (Phronima, Phronimella, Phronimopsis, Hyperoche, Hyperia, Euthemisto, Phrosina, Eupronoe, Simorhynchotus, Calamorhynchus, Steetia, Glossocephalus, Xiphocephalus, Brachyscelus, Platyscelus, Scina, Vibilia). Nach dem sehr wenigen zu urteilen, das bisher von den Ergebnissen der „Pola“-Expedition über die Planktonverhältnisse der Adria und des östlichen Mittelmeeres bekannt geworden ist, hätte man das östliche Mittelmeerbecken als ungemein artenarm betrachten müssen. Die Bearbeitung des „Najade“-Materials wird uns hoffentlich recht bald darüber Aufklärung geben, ob tatsächlich nur ein so kleiner Bruchteil der bunten, westmediterranen Planktonwelt sich in das Ostbecken und die Adria verirrt.

In der kalten Jahreszeit wird das adriatische Küstenwasser am ausgiebigsten von dem aus dem Mittelmeerbecken zuströmenden Hochseewasser verdrängt; daher dürfte in der Adria der Formenreichtum im Winter am größten sein. Wir haben gesehen, wie sich gegen den Sommer zu die echten Hochseeplanktonten immer weiter nach Süden zurückziehen und neritische Planktonten, der Ausbreitung des Küstenwassers entsprechend, an Terrain gewinnen. Ozeanische Planktonten, die im Sommer noch am weitesten im Norden zu finden sind, werden auch im Plankton des Triester Golfes eine verhältnismäßig frühe Schwärmzeit aufweisen. Ein Vergleich der hier gegebenen Verbreitungskarten (Fig. 1, 3, 5) mit dem in meiner "Planktonkunde" veröffentlichten Planktonkalender des Triester Golfes zeigt in der Tat vielfache Ubereinstimmung. Halocypriden sind z. B. im Sommer viel weiter nordwärts vorgedrungen als die koloniebildenden Radiolarien, während um diese Zeit die Copilien schon den Triester Golf erreicht haben können. Dort wurden sie tatsächlich schon im Sommer (Ende Juni bis Anfang Oktober) im Plankton angetroffen; sobald sie verschwunden sind, pflegen Halocypriden aufzutreten (meist im Winterplankton, Dezember bis März). Ist endlich im Frühling das Hochseewasser noch nicht ganz 
vom Küstenwasser verdrängt, dann können in manchen Jahren noch koloniebildende Radiolarien im Triester Golf erscheinen (1902, April bis Juni). Formen endlich, deren Nordgrenze im Frühling und Sommer das Pomobecken ist, kommen offenbar nie in die nördlichste Adria.

Auf Grund der Copepodenausbeute der "Virchow"-Fahrt 1909 konnte das Vorkommen eines typischen Phao- und Knephoplanktons für die Adria nachgewiesen werden. Die „Najade"-Fahrten machten uns, wie wir gesehen, mit Planktonten bekannt, die Lo Bianco zum Scotoplankton (Tiefseefische, Derapoden, Schizopoden etc.) und Pamplankton (Amphipoden) rechnet. Nyctipelagische Benthosformen sind mehrfach auch auf den beiden letzten Terminfahrten von mir beobachtet worden (Cumaceen, Gammariden, Harpacticiden). Nicht nur bei Küstenformen, sondern auch bei typischen Hochseeplanktonten, selbst bei skotoplanktonischen, konnte ein Aufsteigen aus der Tiefe zur Nachtzeit beobachtet werden, und zwar waren es mejst junge Tiere, die dann an der Oberfläche erschienen. Ein (aktives oder passives) Absteigen lebender Phaoplanktonten in größere Tiefen muß, wie ein kleines Experiment, das ich während einer 24stündigen Beobachtung auf der Hochsee anstellte, ausgesprochen phaoplanktonischen Tieren zum Verhängnis werden. Ich versenkte einige eben an der Oberfläche gefangene Evadne und einen Copepoden (Centropages violaceus) in einer mit Müllergaze abgeschlossenen Glastube langsam (innerhalb 5 Minuten) in $150 \mathrm{~m}$ Tiefe. Nach einer Viertelstunde waren alle Tiere noch lebend. Darauf wurden dieselben Individuen in $300 \mathrm{~m}$ Tiefe versenkt; nach einer halben Stunde waren alle Cladoceren abgestorben und nur der Copepode war anscheinend gesund. Da man bei Phaoplanktonten wohl hochgradige Eurythermie und Euryhalie wird annehmen müssen, könnte der mit der Tiefe zunehmende Wasserdruck als Todesursache angenommen werden.

Das sommerliche Hochseeplankton zeichnete sich diesmal durch besondere Individuenarmut aus. Hier einige Beispiele. Uber der tiefsten Stelle der südlichen Adria fanden sich in 140 Liter geschöpftem Oberflächenwasser nur 6 Copepoden, 1 Evadne, 1 koloniebildende Radiolarie. Die gerade für das Sommerplankton des Triester Golfes so charakteristische Meduse Nausithoe punctata fand ich während der ganzen Fahrt nur in einem einzigen Exemplare (A 11, 20-60 m im Pomobecken). ${ }^{1}$ )

Während der ganzen Fahrt wurde ein einziger lebender Delphin

1) Im Vorjahre (N. III) dürfte sie im Sommerplankton häufiger gewesen sein. 
570 A. Steuer, Ergebnisse der VII. Terminfahrt im Sommer 1912 in der Adria.

gesichtet; Haie, Mondfische und Schildkröten aber kamen diesmal gar nicht zur Beobachtung.

Verhältnismäßig planktonreich (namentlich an Phytoplankton) war wieder die adriatische Flachsee, der Quarnero und der Südabfall des Pelagosarückens. Ganz auffallend planktonreich erschien mir der Kanal von Curzola, dessen Planktonreichtum sich noch im östlichen Teil des Pomobeckens bemerkbar machte, während die Küsten weiter südlich bis zur Bocche und anderseits das Inselgebiet vom Kerkaausfluß nordwärts mir wegen seiner Planktonarmut auffiel.

In vertikaler Richtung machte sich eine deutliche Verarmung der Zone etwa zwischen 100 und $200 \mathrm{~m}$ bemerkbar. Im tiefen südlichen Becken dürfte es erst wieder bei ca. $500 \mathrm{~m}$ zu einem etwas reicheren Planktonleben kommen. Das hängt vielleicht mit den hier im kälteren Tiefenwasser mit seiner größeren Viskosität sich anstauenden Leichen der Epiplanktonten zusammen, die dem "Mesoplankton" (Fowler) zur Nahrung dienen. Doch bedürfen diese, nur auf oberflächlichen, während der Fahrt gewonnenen Eindrücken beruhenden Annahmen noch der Uberprüfung durch genaue Messungen.

Einer sehr auffallenden Erscheinung möchte ich noch zum Schlub Erwähnung tun. Im Sommerplankton fehlten diesmal vollständig erwachsene Cymbulien. Während der Sommerfahrten 1909 und 1911 des

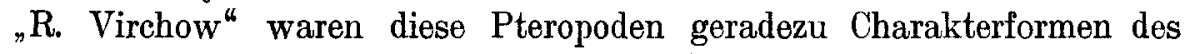
Pomobeckens. Noch im November 1911 (N. IV) wurden sie in größerer Zahl im südlichen Becken gefangen. Im Mai-Juni 1912 (N. VI) fand ich nur noch kleine Individuen. Wir ersehen daraus jedenfalls, daB sich der Lebenszyklus der Planktonten durchaus nicht in jedem Jahre in genau derselben Weise abspielt: Die Cymbulien waren im Sommer 1912 vollkommen von Salpen (darunter die seltene Salpa fusiformis in großer Volkszahl) und besonders den großen Doliolen verdrängt worden. Jenseits der Straße von Otranto schien ihr Plurimum in größeren Tiefen zu liegen, in der Adria selbst dürfte es bis zur Pelagosaschwelle bei etwa $60 \mathrm{~m}$, im Pomobecken bei $20 \mathrm{~m}$ Tiefe gelegen gewesen sein. In größter Dichte schienen sie im zentralen Teil des Pomobeckens vorgekommen zu sein (A 20 östlich Pelagosa und A 13 über der gröBten Tiefe des Pomobeckens). Vielleicht gehen, wịe anderwärts, auch in der Adria derartige Jahresschwankungen im Ablauf des Planktonlebens mit Schwankungen im Wasseraustausch parallel. 